\title{
ARTICULOS
}

CRÍTICA, Revista Hispanoamericana de Filosofia

Vol. XXII, No. 64 (abril 1990): 3-22

\section{ESTRUCTURAS Y REPRESENTACIONES}

ADOLFo GaRcía dE la SIENRA

Instituto de Investigaciones Filosóficas Universidad Nacional Autónoma de México

Las estructuras matemáticas se utilizan en las ciencias naturales y sociales para representar tipos particulares de fenómenos. El objetivo del presente artículo es sentar una base filosófica para discutir esta clase de representación. En la primera parte introduzco un marco ontológico para este propósito y en la segunda discuto un caso particularmente claro de representación, para dar una ilustración de la manera en que considero que el problema de la representación debe ser abordado. Doy por sentado que el lector tiene una buena comprensión del concepto de estructura tal y como este concepto es definido, por ejemplo, por Bourbaki (1968) o Balzer, Moulines y Sneed (1987).

Desde un punto de vista ontológico, las estructuras son entidades abstractas (entes de razbn, como dirían los escolásticos) que pueden ser objeto de investigación matemática. Algunas de estas entidades, por añadidura, representan entes, sistemas o procesos reales. La teoría de la representación es una disciplina filosófica que ambiciona comprender la manera en que las estructuras son portadoras de conocimiento de aspectos o partes del mundo real. El principal problema de la teoría de la representación es dar cuenta de la forma en que la matemática se aplica a la realidad. El presente artículo se halla muy lejos 
de pretender proporcionar una visión completa de los problemas y resultados de esa disciplina; su ambición es sólo hacer un bosquejo de la misma y discutir un caso importante y útil de representación. Por ende, es más un programa que una teoría acabada (aunque en él se encuentren algunos resultados muy sistemáticos).

\section{El marco ontológico}

De acuerdo con la cosmología y la historia natural, la Tierra, los planetas y millones de estrellas eran ya bastante antiguas cuando el homo sapiens comenz6 a maravillarse por el mundo que lo rodeaba. Lo que esto significa es que la mente humana ocupa un lugar más bien modesto en la creación, lejano del lugar de creador del mundo natural. Por el contrario, el hombre siempre ha tratado de dominar los fenómenos naturales para sus propios fines, una tarea cuyo éxito está siempre limitado por la vastedad del mundo. Desde los tiempos en que los hombres se asustaban con el relámpago y el trueno, esa tarea ha sido efectuada a través de un conocimiento creciente de los fenómenos naturales. Originalmente este conocimiento era más bien empírico y asistemático, pero desde el surgimiento de la astronomía en Babilonia, y especialmente en la Grecia clásica con Eudoxo, ese conocimiento se ha vuelto teórico y sistemático. Después de la revolución científica en los siglos diecisiete y dieciocho, han surgido muchas disciplinas científicas más, incluyendo disciplinas que tratan también con fenómenos sociales. Entre otras cosas, estas disciplinas están constituidas por teorías y estructuras conceptuales mediante las cuales los científicos — como antes - intentan obtener el conocimiento de algunas partes o aspectos del mundo real.

El desarrollo de la lógica matemática en el presente siglo - una disciplina que puede ser dividida en teoría de los modelos, de la demostración, de la recursión y de los conjuntosha proporcionado nuevas herramientas para analizar las teorías 
y estructuras conceptuales producidas por las diferentes ciencias, dando así un nuevo sesgo a la teoría de la ciencia, una disciplina fundada por el filósofo y matemático bohemio Bernardo Bolzano con su Wissenschaftslehre en 1837. Desde el punto de vista propugnado aquí, la teoría de la ciencia puede ser definida como la disciplina a cargo de determinar los fundamentos y estructura lógicos de las teorías científicas, así como la manera en que dichas teorías están conectadas con los fenómenos reales con los que tratan. Esta definición de la teoría de la ciencia presupone que hay una manera de referirse al mundo real que es independiente de y, en algún sentido, "previa" al lenguaje de cualquier teoría científica dada. Esta presuposición es verdadera: esa manera es proporcionada por el sistema conceptual y el lenguaje de la philosophia de ente, enriquecido con nombres propios y comunes que refieren a clases naturales, sustancias y accidentes de sustancias. Mediante tal lenguaje podemos ciertamente referirnos a los entes con los que tratan las ciencias, así como formular proposiciones que son verdaderas acerca de tales entes, de una manera independiente del lenguaje técnico de dichas ciencias. La philosophia de ente contiene el aparato conceptual requerido para discutir, por ejemplo, si un objeto considerado por un modelo teórico es un ente o si meramente parece tener unidad individual debido precisamente a la manera en que la mente lo considera; o si un objeto denotado por el lenguaje de una disciplina científica existe de manera real o meramente conceptual -i.e. si es meramente una idealización.

Alguien podría objetar que si tuviéramos una manera independiente de referimos a los entes con los que tratan las ciencias, entonces las estructuras matemáticas que los representan serían redundantes e inútiles. La réplica a esto es que una cosa es referir a algo y otra muy diferente es representarlo matemáticamente. La razón para perseguir una representación matemática de un ser, proceso o sistema real es principalmente penetrar más profundamente en su operación propia, o en sus 
relaciones, y muy frecuentemente obtener mediciones de alguna cantidad en él inherente la cual es requerida para fines teóricos.

La philosophia de ente que tengo en mente - también conocida como ontología o metafísica - fue fundada por Aristóteles unos cinco siglos antes de Cristo, perfeccionada en algunos respectos por los escolásticos y cultivada de manera muy ejemplar y sistemática tanto por Francisco Suarez como por Gottfried Wilhelm Friedrich Leibniz (aunque esto no quiere decir que esté dispuesto a admitir muchas de las suposiciones, más bien barrocas, de este último autor). La philosophia de ente es previa a cualquier ciencia en el sentido de que su lenguaje y aparato conceptual no presuponen el de ninguna ciencia. Esto se debe a dos hechos: (1) su objeto de estudio es el ser, el tópico más general de todos; y (2) el ser es lo primero que se da al entendimiento. Tomo este último enunciado como significando que la estructura básica y la comprensión asistemática del ser se dan en una experiencia que no tiene por qué ser científica. Por ejemplo, la distinción sustancia-accidente, o la distinción cualidad-relación, pueden ser y de hecho fueron establecidas independientemente de toda teoría científica. Ellas son el resultado de una elaboración teórica de experiencias obtenidas principalmente en la transformación de la naturaleza por el hombre a través del trabajo. Esto no significa que no pueda haber retroalimentación de la ciencia a la ontología. Claramente, el concepto de sistema, o el de relación irreductible a cualidades, es un ejemplo de concepto ontologico, no perteneciente al corpus original de la philosophia de ente, fuertemente demandado por las ciencias actuales. Pero la ontología no puede ser reducida a ninguna ciencia y tiene que ser cultivada con sus propios métodos y procedimientos.

Algunos principios de la philosophia de ente, que son pertinentes para los propósitos de este trabajo, se hallan entre los siguientes: 
(1) Hay una multiplicidad de entes, no todos los cuales son contenidos de alguna mente.

(2) El conjunto de todos los entes es dividido en dos clases fundamentales: (i) sustancias, y (ii) accidentes. Los primeros no requieren de otros entes como sustratos, mientras que los segundos inhieren en los primeros.

(3) Algunas sustancias son materiales, es decir, están compuestas de materia y forma, siendo así susceptibles de generación y corrupción.

(4) Toda sustancia material posee accidentes.

(5) El conjunto de los accidentes se divide en categorías, no mutuamente disjuntas, entre las que distinguimos las siguientes: (i) cantidad, (ii) cualidad, (iii) relación, (iv) estado, (v) acción.

Estos no son más que unos pocos de los principios de la philosophia de ente. Una discusión de todos ellos bastante completa, si bien anacrónica, se halla en las Disputaciones metafísicas de Suárez ${ }^{1}$ y, desde luego, en los textos clásicos del Filósofo.

El hilo conductor de esta investigación será el problema de la aplicación de las matemáticas a la realidad. El concepto mediante el cual intentaré explicar esta aplicación es el de medición fundamental. Para introducir este concepto, definiremos una estructura ontológica como una estructura de alguna especie, de la forma $\left\langle A, R_{1}, \ldots, R_{n}\right\rangle$, que satisface ciertos enunciados ontológicos $\phi_{1}, \ldots, \phi_{s}$, tal que los elementos de $A$ son entes reales y $\operatorname{los} R_{i}(i=1, \ldots, n)$ son relaciones conjuntistas entre los elementos de $A$ que representan relaciones u operaciones reales entre los mismos elementos. Si $r$ es una relación real entre entes, decimos que $R$ representa $r$ sys $R$ es un conjunto de tuplos de entes relacionados por $r$ o, más precisamente, si

1 Un exponente contemporáneo de esta ontología es Gracia (1988). 
' $F x_{1}, \cdots, x_{k}$ ' expresa el hecho de que los entes $x_{1}, \ldots, x_{k}$ están relacionados por $r$, decimos que $R$ representa $r$ sys el hecho de que $\left\langle x_{1}, \ldots, x_{k}\right\rangle \in R$ implica que $F x_{1}, \cdots, x_{k}$.

Una estructura numérica es una estructura como la anterior, sólo que el conjunto subyacente $A$ es un conjunto de números reales. Una medición fundamental de las cantidades, operaciones o relaciones reales $r_{1}, \ldots, r_{n}$ de o entre los entes $x_{1}, \ldots, x_{k}$ es un homomorfismo $\varphi$ de la estructura $\mathfrak{A}=\left\langle A, R_{1}, \ldots, R_{n}\right\rangle$ en una estructura numérica $\mathfrak{B}=\left\langle B, S_{1}, \ldots, S_{n}\right\rangle$, donde $A=$ $\left\{x_{1}, \ldots, x_{k}\right\}$ y $R_{i}$ representa a $r_{i}(1 \leq i \leq n)$. Cuando tal homomorfismo existe, decimos que $\mathfrak{B}$ representa $\mathfrak{A}$, y también que $\varphi$ representa las relaciones $R_{i}$. Así, la función $\varphi: A \rightarrow B$ representa la relación $R_{i}$ (y así, indirectamente, también la relación $\left.r_{i}\right)$ sys, para todo $\left\langle x_{1}, \ldots, x_{m}\right\rangle \in R_{i}$,

$$
\left\langle x_{1}, \ldots, x_{m}\right\rangle \in R_{i} \Leftrightarrow\left\langle\varphi\left(x_{1}\right), \ldots, \varphi\left(x_{m}\right)\right\rangle \in S_{i} .
$$

Un teorema de representación de una estructura ontológica $\mathfrak{A}$ es un enunciado que afirma la existencia de una función representando las relaciones de $\mathfrak{A}$, y también estableciendo hasta qué punto es única esa función, que puede ser derivado lógicamente a partir de los enunciados ontológicos $\phi_{1}, \ldots, \phi_{s}$. La cláusula del teorema de representación que afirma la existencia de la representación es llamada la parte existencial; la que expresa el grado de unicidad es llamada la parte de unicidad.

El papel de la ontología en el establecimiento de un teorema de representación consiste en proporcionar el aparato conceptual requerido para discutir y formular los axiomas ontolbgicos $\phi_{1}, \ldots, \phi_{s}$. Será beneficioso ilustrar este papel con un ejemplo. Ese es el objetivo de la siguiente sección.

\section{Un caso de representacion}

La situación es la siguiente. Hay una sustancia material $\longrightarrow$ digamos una viga de madera - que tiene la forma de un para- 
lelepípedo. La altura de cualquier paralelepípedo es la longitud de cualquiera de los segmentos ortogonales a sus bases y encerrados por esas bases. De acuerdo con Francisco Suárez, quien sigue al Filosofo en este respecto, ${ }^{2}$ estos segmentos no son imaginarios, dado que son seres reales en la categoría de la cantidad, inherentes en la viga dada. Nuestra tarea es darle sentido ontológico a la medición de las longitudes de estos segmentos - que llamaremos 'segmentos principales'- y a la de sus subsegmentos.

Por supuesto, no cualquier asignación de números a los segmentos contaría como una medición de sus longitudes. La primera condición que una medición tal tiene que cumplir es que debe asignar el mismo número a segmentos de la misma longitud, y otro requisito es que si un segmento puede ser dividido en dos segmentos $x, y$, entonces los números asignados al segmento y a sus partes $x, y$ debe ser tal que la suma de los números asignados respectivamente $\mathrm{a} x, y$ tiene que ser igual al número asignado al segmento completo, i.e. la medición tiene que ser extensiva. Una cuestión que surge naturalmente es qué tan finamente tiene que estar granulado el segmento principal, cuáles son los segmentos más pequeños en los que puede ser dividido el segmento, o si la división puede continuar al infinito. Esto plantea el viejo problema metafísico de la composición del continuo, un problema que fue caracterizado por Leibniz como uno de los laberintos de la mente humana. ${ }^{3}$ Este profundo problema metafísico tiene una incidencia directa en la elección de los axiomas que garantizan la existencia y unicidad de la medición. Krantz et al. (1971) introdujeron un Axioma de regularidad para la medición extensiva que sólo puede ser interpretado de dos maneras: (i) los segmentos pueden ser infinitamente divididos (éste es el punto de vista de Leibniz), o

2 Ver Disputación XL, Parte VI, $\S 5$.

3 Cf. Brown (1984), p. 153, n. 12. El otro "laberinto" es el problema de reconciliar la presciencia de Dios con el libre albedrío humano. 
(ii) hay una subdivisión más pequeña $d$ tal que la longitud de cualquier otra subdivisión es un múltiplo de la de $d$ (esta es una versión del punto de vista opuesto). En general, el punto de vista opuesto es precisamente que los segmentos pueden ser divididos en un número finito de partes más pequeñas, siendo sus longitudes no necesariamente múltiplos de la longitud de la parte más pequeña de ellos. De acuerdo con la física contemporánea, los cuerpos no pueden ser divididos ad infinitum, de modo que apoya más bien el punto de vista finitista.

La cuestión que surge ahora es si la estructura mereológica de cualquier segmento garantiza la existencia de mediciones de longitud, suponiendo que el punto de vista finitista es verdadero. Claro, puesto que la medición de longitud consiste en comparar cualquier segmento con un segmento común tomado como unidad, i.e. en determinar "cuántas réplicas concatenadas" de esta unidad son equivalentes a cualquier longitud determinada, si no hay parte o segmento más pequeño tal que todo otro segmento es un múltiplo de esa parte más pequeña, entonces no es posible ninguna medición. La única manera de medir los segmentos en los cuales puede ser dividida la altura del segmento principal es entonces traer una unidad de fuera del segmento tal que todos aquellos segmentos son múltiplos de dicha unidad. Nótese que esto presupone (i) qué hay otra sustancia material tal que posee un segmento de línea del tipo requerido, y (ii) que los segmentos en los cuales la altura de la viga puede ser dividida son conmensurables.

En el caso de las vigas, consideraciones de homogeneidad hacen plausible que la rebanada a lo ancho más delgada tenga la misma anchura por doquier, de modo que en este caso el Axioma de regularidad es vendadero con la interpretación de que hay una subdivisión más pequeña $d$ tal que la longitud de cualquier otra subdivisión es un múltiplo entero de la de $d$. Observe que el Axioma de regularidad que introduciremos también se puede interpretar como una consecuencia de la concepción leibniziana del continuo. Obviamente, la representa- 
ción requerida puede ser construida meramente asignándole el número 1 a cualquier subdivisión más pequeña $d$ y el número $k$ a un segmento que sea equivalente a " $k$ réplicas concatenadas de $d$ ". Pero ésta es una manera operacionalista descuidada de describir la construcción. Un tratamiento filosófico adecuado requiere la introducción de herramientas conceptuales más precisas.

Considere al conjunto $X$ que tiene como elementos un segmento principal particular, ortogonal a las bases de la viga y determinado por esas bases, así como todas sus partes potenciales, i.e. los segmentos en que puede ser dividido hasta los segmentos más pequeños. Si $x, y$ son cualesquiera elementos $\operatorname{de} X$, escribimos $x \succsim y$ sys la magnitud de $x$ es mayor o igual que la magnitud de $y$. Observe que esta relación es independiente de que se efectúe realmente la comparación entre segmentos por un agente y, por ende, no debe ser concebida operacionalistamente. Escogemos ahora una dirección en la viga, por ejemplo con respecto a las manos, y designamos una derecha y una izquierda en la viga (a lo largo). Introducimos ahora un conjunto $Y$ de pares $(y, z) \in X \times X$ como sigue: $\mathrm{El}$ par $(y, z)$ está en $Y$ sys hay un segmento $x \in X$ tal que $x$ puede ser dividido en $y$ y $z$, y $y$ es un subsegmento de $x$ a la izquierda de $z$; en este caso escribimos $x=y \oplus z$. Observe que el hecho de que hayamos escogido determinar la dirección del segmento con respecto a las manos no hace a la dirección una entidad subjetiva. Una dirección es una ondenación real entre las partes de un cuerpo; en el caso presente, hay dos ordenaciones entre las partes del segmento, cada una determinada por un extremo de la viga, el cual es el primer elemento en el orden correspondiente. Al elegir una derecha y una izquienda en la viga sólo estamos escogiendo una de estas dos ordenaciones preexistentes. La siguiente definición introduce axiomas que son conjuntamente suficientes para demostrar el teorema de representación requerido. Discuto su significado y justificación metafísicos más abajo. 
DEFINICIÓN 1: $\langle X, \succsim, Y, \oplus\rangle$ es una estructura extensiva aristotélica sys se cumplen los siguientes axiomas:

(A1) $\langle X, \succsim\rangle$ es un orden débil

(A2) (Congruencia) Si están definidos $x \oplus y$ y $z \oplus w$, y $x \sim z$, $y \sim w$, entonces $x \oplus y \sim z \oplus w$

(A3) (Dominancia) Si $(x, y) \in Y, x \succsim z$ y $y \succsim w$, entonces hay $u, v \in X$ tales que $(u, v) \in Y$ y $u \sim z, v \sim w$. Más aún, $x \oplus y \succsim u \oplus v$

(A4) (Descomposición) Si $(x, y) \in Y$ y $x \oplus y \sim z$, entonces existen $u, v \in X$ tales que $(u, v) \in Y, u \sim x, v \sim y$ y $z=u \oplus v$

(A5) (Asociatividad) $(x, y) \in Y$ y $(x \oplus y, z) \in Y$ sys $(y, z) \in Y$ y $(x, y \oplus z) \in Y$; y cuando ambas condiciones son el caso

$$
(x \oplus y) \oplus z=x \oplus(y \oplus z)
$$

(A6) (Positividad) Si $(x, y) \in Y$, entonces $x \oplus y \succ x$

(A7) (Regularidad) Si $x \succ y$, entonces existen $z, w, u \in X$ tales que $z \sim x, w \sim y,(w, u) \in Y$ y $z \succsim w \oplus u$

(A8) (Axioma arquimediano) Toda secuencia estándar estrictamente acotada es finita. Decimos que

$$
x_{1}, \ldots, x_{n}, \ldots
$$

es una secuencia estándar sys hay una secuencia

$$
y_{1}, \ldots, y_{n}, \ldots
$$

tal que $y_{i} \sim y_{j}$ con $\left(y_{i}, y_{i+1}\right) \in Y(i, j=1, \ldots, n, \ldots)$ y, más aún, hay otra secuencia

$$
z_{1}, \ldots, z_{n}, \ldots
$$


definida por $z_{1}=y_{1}, z_{n}=z_{1} \oplus z_{n-1}$, con $x_{k} \sim z_{k}$; es estrictamente acotada sys hay un $x \in X$ tal que $x \succ x_{n}$ para todo $x_{n}$ en la secuencia.

Como ya es usual, ' $x \sim y$ ' es una abreviatura de ' $x \succsim y$ y $y \succsim x$ '; mientras que ' $x \succ y$ ' es una abreviatura de ' $x \succsim y$ y no $y \succsim x^{\prime}$

El axioma (Al) afirma que dos segmentos cualesquiera en $X$ son comparados por la relación $\succsim$, i.e. que de cualquier par $x, y \in X$ o bien la longitud de $x$ es mayor o igual que la longitud de $y$ o viceversa. También, que la relación $\succsim$ es transitiva. Se puede ver que el axioma es metafísicamente verdadero.

(A2) afirma la congruencia de dos segmentos cualesquiera cuyas partes en las que son divisibles por dos son ellas respectivamente congruentes.

(A3) afirma que si hay un segmento dividido en dos, cuyas partes dominan respectivamente sendos segmentos, entonces hay otro segmento también divisible en dos, cuyas partes son respectivamente congruentes a los segmentos dominados, y que es él mismo dominado por el segmento original más largo. Un poco de reflexión espacial revela que esto es correcto.

(A4) afirma que si un segmento es congruente con un segmento divisible en dos partes, entonces el primer segmento puede ser dividido en dos partes que son congruentes con las partes del segundo segmento. Es fácil ver que el axioma es verdadero.

(A5) es obvio, pues no importa el orden en que un segmento es dividido.

(A6) es también obvio, pues la longitud de un segmento es siempre mayor que la longitud de cualquiera de sus segmentos propios.

(A7) es el Axioma de regularidad mencionado anteriormente en conexión con el problema de la composición del continuo. Lejos de ser obviamente verdadero, expresa una solución determinada al enredo metafísico de la naturaleza del continuo. EI 
axioma es verdadero, sin embargo, si los segmentos de líneas inherentes en los cuerpos son continuos en el sentido adoptado por Leibniz y Aristóteles (en La flsica, Libro VI, 231 20 $231^{\text {b}} 21$ ), o si es el caso que están compuestos de un número finito de subsegmentos congruentes como se discutió arriba.

El sentido del axioma (A8) es que si tenemos una secuencia de segmentos, la longitud de cada término de la secuencia siendo mayor que el término previo exactamente por la misma diferencia que la de cualquier otro par de términos consecutivos, y todos los términos de la secuencia están estrictamente dominados por el mismo segmento, entonces la secuencia es finita. A grandes rasgos, esto afirma la inexistencia de segmentos de longitud infinitesimal, i.e. de segmentos tales que ninguna adición finita de los mismos - no importa cuán larga — jamás sobrepasará la longitud de un segmento finito dado. El axioma parece ser verdadero de longitudes reales.

Estas aserciones metafísicas son suficientes para demostrar el siguiente teorema.

TEOREMA: (Teorema de representación). Sea

$$
\langle X, \succsim, Y, \oplus\rangle
$$

una estructura extensiva aristotélica. Entonces existe una función $\varphi: X \rightarrow \Re$ tal que, para todo $x, y \in X$,

$$
x \succsim y \quad \text { sys } \quad \varphi(x) \geq \varphi(y)
$$

$$
\begin{array}{ll}
\text { si } \quad(x, y) \in Y, & \text { entonces } \\
& \varphi(x \oplus y)=\varphi(x)+\varphi(y)
\end{array}
$$

Si otra función $\varphi^{\prime}$ satisface (i) $y$ (ii), entonces existe un $\alpha>0$ tal que, para todo $x \in X$ no maximal, $\varphi^{\prime}(x)=\alpha \varphi(x)$.

La demostración del teorema es complicada y requiere la construcción de otra estructura, así como la demostración de 
varios lemas concernientes a esta otra estructura. Para construirla, introduciremos la siguiente definición.

DEFINICIÓN 2: $\operatorname{Si}\langle X, \succsim, Y, \oplus\rangle$ es una estructura extensiva aristotélica, construimos el conjunto $Z$ de todos los pares $(x, y)$ $\in X \times X$ tales que hay $z, w \in X \operatorname{con} z \sim x, w \sim y$ y $(z, w) \in Y$. Si $(z, w) \in Y$, decimos que $z \oplus$ w está definido.

La nueva estructura requerida se construye como sigue. Será la estructura $\langle A, \succsim, B, \circ\rangle$ tal que $A$ es el conjunto cociente $X / \sim$ of $X$ con respecto a $\sim$, $\succsim$ es la relación dada por

$$
[x] \succsim[y] \text { sys } x \succsim y,
$$

$B$ es el conjunto $\{([x],[y]):(x, y) \in Z\}$, y o es la operación que asigna a cada par $([x],[y])$ de elementos de $B$ el elemento $[x] \circ[y]=[z \oplus w]$, para algún $z \sim x$ y $w \sim y$. Observe que $\sim$ en estas últimas dos expresiones es la relación sobre $X$; usaré los mismos símbolos $\succsim, \succ \mathrm{y} \sim$ en ambas estructuras, pues el contexto habrá de evitar cualquier posibilidad de confusión. La estrategia de la demostración del teorema de representación consiste en mostrar que $\langle A, \succsim, B, \circ\rangle$ es un semigrupo local, regular, positivo y ordenado. El Lema 1 abajo establece que la relación $\succsim$ y la operación o están bien definidas. Los lemas restantes establecen que la estructura satisface las condiciones definitorias de los semigrupos recién mencionados. ${ }^{4}$

LEMA 1: Tanto $\succsim$ como o están bien definidas.

Demostración: Antes que nada, queremos mostrar que si $[x]$, [y] son elementos de $B$ tales que $[x] \succsim[y]$, y $x \sim x^{\prime}, y \sim y^{\prime}$, entonces $\left[x^{\prime}\right] \succsim\left[y^{\prime}\right]$. Pero esto está claro porque las suposiciones dadas implican que $x^{\prime} \succsim y^{\prime}$, por (Al).

En seguida queremos establecer que el resultado de la operación o no depende de la particular elección de los elementos de la clase de equivalencia, i.e queremos probar que si

4 Para una definición de semigrupo local, regular, positivo y ordenado, véase Krantz et al. (1971), p. 44. 
$[x],[y]$ son clases tales que $([x],[y]) \in B, \mathrm{y} x^{\prime}, y^{\prime}$ son elementos de $X$ tales que $x^{\prime} \sim x, y^{\prime} \sim y$, entonces $\left(\left[x^{\prime}\right],\left[y^{\prime}\right]\right) \in B$ y $[x] \circ[y]=\left[x^{\prime}\right] \circ\left[y^{\prime}\right]$. Las suposiciones dadas implican que hay elementos $z, w, z^{\prime}, w^{\prime} \in X$ tales que $z \sim x, w \sim y, z^{\prime} \sim x^{\prime}$, $w^{\prime} \sim y^{\prime}, z \oplus w$ y $z^{\prime} \oplus w^{\prime}$ están definidos, $[z \oplus w]=[x] \circ[y]$ y $\left[z^{\prime} \oplus w^{\prime}\right]=\left[x^{\prime}\right]$ o [y $\left.y^{\prime}\right]$. Por (A2), tenemos que $[z \oplus w]=\left[z^{\prime} \oplus w^{\prime}\right]$ $\mathrm{y}$ así se sigue el resultado deseado.

LEMA 2: $\langle A, \succsim\rangle$ es un orden simple.

Demostración: Para cualesquiera $[x],[y] \in A$, o bien $x \succsim y$ o bien $y \succsim x$ (por A1), lo que implica que $\succsim$ es conectada en $A$. Si $[x] \succsim[y]$ y $[y] \succsim[x]$, entonces $x \succsim y$ y $y \succsim x$, i.e. $x \sim y$, de donde se sigue que $[x]=[y]$. Finalmente, si $[x] \succsim[y]$ y $[y] \succsim[z]$, entonces $x \succsim y$ y $y \succsim z$, lo que implica que $x \succsim z$ (por Al) y así que $[x] \succsim[z]$.

LEMA 3: $S i([x],[y]) \in B, y[x] \succsim[z],[y] \succsim[w]$, entonces $([z],[w]) \in B$.

Demostración: Puesto que $([x],[y]) \in B$, se sigue que hay $u, v$ con $u \sim x, v \sim y$ y $u \oplus v$ definidos. Se sigue que $u \succsim z$ y $v \succsim w$, y así que hay $u^{\prime}, v^{\prime}$ con $u^{\prime} \sim z, v^{\prime} \sim w$ y $u^{\prime} \oplus v^{\prime}$ definidos (A3). Esto muestra que $(z, w) \in Z$ y así que $([z],[w]) \in B$.

LEMA 4: $S i([z],[x]) \in B y[x] \succsim[y]$, entonces $([z],[y]) \in B y$ $[z] \circ[x] \succsim[z] \circ[y]$

Demostración: Por el Lema 3, $([z],[y]) \in B$. La conclusión deseada se deduce de (A3).

LEMA 5: $S i([x],[z]) \in B y[x] \succsim[y]$, entonces $([y],[z]) \in B y$ $[x] \circ[z] \succsim[y] \circ[z]$.

Demostración: Por Lema 3, $([y],[z]) \in B$. La conclusión deseada se sigue de (A3). 
Por alguna curiosa razón, la demostración de una de las propiedades aparentemente más simples de una operación binaria, la asociatividad, es más bien complicada.

LEMA 6: $([x],[y]) \in B y([x] \circ[y],[z]) \in B$ sys $([y],[z]) \in B$ $y([x],[y] \circ[z]) \in B ; y$ cuando ambas condiciones se cumplen, $([x] \circ[y]) \circ[z]=[x] \circ([y] \circ[z])$.

Demostración: Para establecer el condicional, suponga primero que $([x],[y]) \in B$ y $([x] \circ[y],[z]) \in B$. Entonces hay $x^{\prime}, y^{\prime}$, $u, z^{\prime}$ tales que $x^{\prime} \sim x, y^{\prime} \sim y,[u]=[x] \circ[y], z^{\prime} \sim z$ y $\left(x^{\prime}, y^{\prime}\right) \in Y,\left(u, z^{\prime}\right) \in Y$. También hay $x^{\prime \prime}, y^{\prime \prime}$ tales que $[u]=$ $[x] \circ[y]=\left[x^{\prime \prime} \oplus y^{\prime \prime}\right]$ con $x^{\prime \prime} \sim x$ y $y^{\prime \prime} \sim y$. Es inmediato que $u \sim x^{\prime \prime} \oplus y^{\prime \prime}$ y así (por A4) hay $x^{\prime \prime \prime}, y^{\prime \prime \prime}$ tales que $x^{\prime \prime \prime} \sim$ $x^{\prime \prime}, y^{\prime \prime \prime} \sim y^{\prime \prime},\left(x^{\prime \prime \prime}, y^{\prime \prime \prime}\right) \in Y$ y $u=x^{\prime \prime \prime} \oplus y^{\prime \prime \prime}$. Así, tenemos tanto $\left(x^{\prime \prime \prime}, y^{\prime \prime \prime}\right) \in Y$ como $\left(x^{\prime \prime \prime} \oplus y^{\prime \prime \prime}, z^{\prime}\right) \in Y$. Luego (por A5), $\left(\gamma^{\prime \prime \prime}, z^{\prime}\right),\left(x^{\prime \prime \prime}, y^{\prime \prime \prime} \oplus z^{\prime}\right) \in Y \subseteq Z$ y por lo tanto, puesto que $[x]=\left[x^{\prime \prime \prime}\right],[y]=\left[y^{\prime \prime \prime}\right]$ y $[y] \circ[z]=\left[y^{\prime \prime \prime} \oplus z^{\prime}\right]$, tenemos que $([y],[z]) \in B$ y $([x],[y] \circ[z]) \in B$. La recíproca se prueba de modo análogo.

Suponga ahora que ambas condiciones se dan. Por (A4), para cualquier $x, y, z \in X$ hay $x^{\prime}, y^{\prime}$ y $z^{\prime}$ tales que $x^{\prime} \sim x, y^{\prime} \sim y$, $z^{\prime} \sim z$ y

$$
([x] \circ[y]) \circ[z]=\left[\left(x^{\prime} \oplus y^{\prime}\right) \oplus z^{\prime}\right] .
$$

Así, por (A5),

$$
\begin{aligned}
([x] \circ[y]) \circ[z] & =\left[x^{\prime} \oplus\left(y^{\prime} \oplus z^{\prime}\right)\right] \\
& =[x] \circ\left[y^{\prime} \oplus z^{\prime}\right] \\
& =[x] \circ([y] \circ[z]) .
\end{aligned}
$$

LEMA 7: Si $([x],[y]) \in B$, entonces $[x] \circ[y] \succ[x]$.

Demostración: Suponga que $([x],[y]) \in B$. Entonces $(x, y) \in Z$ y así $[x] \circ[y]=[z \oplus w]$ para $z \sim x, w \sim y$. Por (A6), $z \oplus w \succ z$ $y$ así $[x] \circ[y]=[z \oplus w] \succ[z]=[x]$. 
LEMA 8: $S i[x] \succ[y]$, entonces hay $[z] \in A$ tal que $([y],[z]) \in B$ $y[x] \succsim[y] \circ[z]$.

Demostración: Suponga que $[x] \succ[y]$. Entonces $x \succ y$ y así (por A7) hay $u, v, z \in X$ con $u \sim x, v \sim y(v, z) \in Y$ y $u \succsim v \oplus z$. Por ende, $(\gamma, z) \in Z$ y

$$
[x]=[u] \succsim[v \oplus z]=[y] \circ[z] .
$$

Para cualquier $[x] \in A$ definimos la expresión $n[x]$ como sigue. Si $y_{1} \oplus \cdots \oplus y_{n}$ está definida y $x \sim y_{i}$ para cualquier $i$ $(1 \leq i \leq n)$, hacemos $n[x]=\left[y_{1} \oplus \cdots \oplus y_{n}\right]$ y decimos que $n[x]$ está definida. Hacemos también que $N_{[x]}$ sea el conjunto $\{n \in N: n[x]$ está definido $\}$.

LEMA 9: $\left\{n: n \in N_{[x]}\right.$ y $\left.[y] \succ n[x]\right\}$ es un conjunto finito.

Demostración: Por definición, para todo $n \in N_{[x]}$ hay $y_{1}, \ldots, y_{n} \in X$ con $y_{i} \sim x$ para todo $i=1, \ldots, n$, tal que

$$
n[x]=\left[y_{1} \oplus \cdots \oplus y_{n}\right]
$$

Así, a la secuencia $1[x], 2[x], \ldots, n[x], \ldots$ (que está definida porque $m \in N_{[x]}$ para todo $m<n$ ) corresponde biunívocamente una secuencia estándar $x_{1}, x_{2}, \ldots, x_{n}, \ldots$ en $X$. La condición $[y]$ $\succ n[x]$ implica que esta secuencia es estrictamente acotada $y$, por lo tanto (por A8), es finita, lo que a su vez implica que el conjunto $\left\{n: n \in N_{[x]}\right.$ y $\left.[y] \succ n[x]\right\}$ es finito.

Procederemos ahora a la demostración del resultado principal, utilizando los anteriores lemas preparatorios.

Demostración del Teorema de representación: Sea

$$
\langle X, \succsim, Y, \oplus\rangle
$$

una estructura extensiva aristotélica, y defínase una nueva estructura $\langle A, \succsim, B$, o $\rangle$ como sigue. Sea $A=X / \sim$ el conjunto 
cociente de $X$ con respecto a $\sim$; para cualquier $[x],[y] \in A$, sea $[x] \succsim[y]$ sys $x \succsim y$; sea $B=\{([x],[y]):(x, y) \in Z\}$; finalmente, si $([x],[y]) \in B$ entonces $[x] \circ[y]=[z \oplus w]$ para $z \sim x$ y $w \sim y$. Por los lemas $1-9,\langle A, \succsim, B$, o) es un semigrupo local, regular, positivo, ordenado y arquimediano. Así por el Teorema 2.4 en Krantz et al. (1971), p. 45, hay una función $\psi$ de $A$ en $\Re^{+}$tal que, para todo $[x],[y] \in A$,

$$
[x] \succsim[y] \quad \text { sys } \quad \psi([x]) \geq \psi([y])
$$

y

(ii) si $([x],[y]) \in B$, entonces

$$
\psi([x] \circ[y])=\psi([x])+\psi([y]) .
$$

Más aún, si $\psi$ y $\psi^{\prime}$ son dos funciones cualesquiera de $A$ a $\Re^{+}$ que satisfacen las condiciones (i) y (ii), entonces existe $\alpha>0$ tal que para cualquier $[x] \in A$ no maximal,

$$
\psi^{\prime}([x])=\alpha \psi([x]) \text {. }
$$

Sea $\varphi$ la función de $X$ a $\Re^{+}$definida como sigue: Si $x \in X$, sea $\varphi(x)=\psi([x])$. Entonces tenemos

$$
\begin{array}{lll}
x \succsim y & \text { sys } & {[x] \succsim[y]} \\
& \text { sys } & \psi([x]) \geq \psi([y]) \\
& \text { sys } & \varphi(x) \geq \varphi(y) .
\end{array}
$$

también, si $(x, y) \in Y$, entonces $([x],[y]) \in B$ y

$$
\begin{aligned}
\varphi(x \oplus y) & =\psi([x \oplus y]) \\
& =\psi([x] \circ[y]) \\
& =\psi([x])+\psi([y]) \\
& =\varphi(x)+\varphi(y) .
\end{aligned}
$$

Suponga ahora que $\varphi^{\prime}$ es otra función que satisface las condiciones (i) y (ii) del teorema. Sea $\psi^{\prime}$ la función de $A$ a $\Re^{+}$tal 
que $\psi^{\prime}([x])=\varphi^{\prime}(x)$. Entonces es fácil mostrar que $\psi^{\prime}$ es una representación de $\langle A, \succsim, B, 0\rangle$ diferente de $\psi$. Observe que $x$ es maximal en $X$ sys $[x]$ es maximal en $A$. Por ende, hay un $\alpha$ positivo tal que, para todo $x$ no maximal,

$$
\begin{aligned}
\varphi^{\prime}(x) & =\psi^{\prime}([x]) \\
& =\alpha \psi([x]) \\
& =\alpha \varphi(x) .
\end{aligned}
$$

El teorema de representación queda así demostrado.

Las demostraciones anteriores han ilustrado, en un caso clásico muy importante, un procedimiento típico de la teoría de la representación que propugno aquí. ¿Es posible tratar todos los conceptos cuantitativos usados en las ciencias de la misma manera que el caso anterior, es decir, en términos de representaciones de sustancias, accidentes o procesos reales? ¿Es posible dar un tratamiento unificado para todos los casos de representación? Éstos son, sin duda alguna, problemas centrales de la teoría de la ciencia que tienen que ver con el problema de la transición de los que Georgescu-Roegen (1971) ha llamado 'conceptos dialécticos' a lo que él mismo ha llamado 'conceptos aritmomórficos', i.e. la transición de conceptos metafísicos más o menos vagos y difusos, si bien indispensables para pensar la realidad, a conc sptos rígidos y precisos, como lo son los conceptos matemáticos.

\section{BIBLIOGRAFIA}

Balzer, W., Moulines, C. U., Sneed, J. D., An Architectonic for Science, D. Reidel, Dordrecht, 1987.

Bourbaki, N. (pseud.), Elements of Mathematics: Theory of Sets, Addison-Wesley, Reading, 1968.

Brown, S., Leibniz, University of Minnesota Press, Minneapolis, 1984. 
Georgescu-Roegen, N., The Entropy Law and the Economic Process, Harvard University Press, Cambridge, 1971.

Gracia, J. J. E., Individuality. An Essay on the Foundations of Metaphysics, SUNY Press, Albany, 1988.

Krantz, D. H., R. D. Luce, P. Suppes, A. Tversky, Foundations of Measurement I, Academic Press, New York, 1971.

Suárez, F., Disputaciones metafisicas, Gredos, Madrid, 1960.

Rocibido: 25 abril 1989. 


\section{SUMMARY}

The aim of the present paper is to set a philosophical basis in order to discuss the type of representation that holds between mathematical structures and those aspects of the real world which they represent. It is maintained that an actualized version of Aristotelian metaphysics is suited for this purpose. The connection between the abstract, rigid concepts of mathematics, and the concepts of metaphysics is attempted through the concept of a fundamental measurement. The existence and degree of uniqueness of a fundamental measurement is established as a representation theorem asserting the existence of a homomorphism from what I call an ontological structure into a numerical one. An ontological structure contains as elements real beings, and its relations represent -in a sense made precise thereof - real relations among these beings. The role of metaphysics in the establishment of a representation theorem is to provide the conceptual apparatus required to discuss and formulate the ontological axioms required to derive the theorem. The paper contains a very complete example of a fundamental measurement in the sense described, namely, the measurement of the height of a physical parallelepiped and that of its potential parts. 